\title{
Author Correction: Induced unconventional superconductivity on the surface states of $\mathrm{Bi}_{2} \mathrm{Te}_{3}$ topological insulator
}

\author{
Sophie Charpentier', Luca Galletti (10 1, Gunta Kunakova1,2, Riccardo Arpaia (10 1, Yuxin Song (1) 1,3, Reza Baghdadi', \\ Shu Min Wang ${ }^{1,3}$, Alexei Kalaboukhov1, Eva Olsson (10 4, Francesco Tafuri5 ${ }^{5,6}$, Dmitry Golubev7 , Jacob Linder ${ }^{8}$, \\ Thilo Bauch $^{1} \&$ Floriana Lombardi (i) ${ }^{1}$
}

Correction to: Nature Communications https://doi.org/10.1038/s41467-017-02069-z, published online 08 December 2017

The original version of this Article omitted the following from the Acknowledgements:

"This work was partly supported by the Research Council of Norway through its Centres of Excellence funding scheme, project number 262633, QuSpin."

This has now been corrected in both the PDF and HTML versions of the article.

Published online: 05 January 2018

\begin{abstract}
reproduction in any medium or format, as long as you give appropriate credit to the original author(s) and the source, provide a link to the Creative Commons license, and indicate if changes were made. The images or other third party material in this article are included in the article's Creative Commonslicense, unless indicated otherwise in a credit line to the material. If material is not included in the article'sCreative Commons license and your intended use is not permitted by statutory regulation or exceeds the permitted use,
\end{abstract} you will need to obtain permission directly from the copyright holder. To view a copy of this license, visit http://creativecommons.org/licenses/by/4.0/.

(c) The Author(s) 2018

\footnotetext{
${ }^{1}$ Department of Microtechnology and Nanoscience, Chalmers University of Technology, SE-41296 Göteborg, Sweden. ${ }^{2}$ Institute of Chemical Physics, University of Latvia, 19 Raina Boulevard, LV-1586 Riga, Latvia. ${ }^{3}$ Shanghai Institute of Microsystem and Information Technology, Chinese Academy of Sciences, 865 Changning Road, Shanghai CN-200050, China. ${ }^{4}$ Department of Applied Physics, Chalmers University of Technology, SE-41296 Göteborg, Sweden. ${ }^{5}$ Dipartimento di Fisica E. Pancini, Università di Napoli Federico II, IT-80126 Napoli, Italy. ${ }^{6}$ CNR-SPIN Institute of Superconductors, Innovative Materials and Devices, IT-80125 Napoli, Italy. ${ }^{7}$ Department of Applied Physics, Aalto University School of Science, P.O. Box 13500, Aalto FI-00076, Finland. ${ }^{8}$ Department of Physics, QuSpin Center of Excellence, Norwegian University of Science and Technology, N-7491 Trondheim, Norway. S. Charpentier and L. Galletti contributed equally to this work. Correspondence and requests for materials should be addressed to F.L. (email: floriana.lombardi@chalmers.se)
} 